\title{
EZ (EASY?) CANIS MAJORIS
}

\author{
C. ROBERT ${ }^{1}$, A. F. J. MOFFAT ${ }^{1}$, L. DRISSEN ${ }^{2}$, V. S. NIEMELA ${ }^{3}$, P. BARRETT ${ }^{4}$, \\ W. SEGGEWISS ${ }^{5}$, and R. LAMONTAGNE ${ }^{1}$ \\ ${ }^{1}$ Université de Montréal, Montréal, Canada; ${ }^{2}$ Space Telescope Science Institute, \\ Baltimore, U.S.A.; ${ }^{3}$ IAFE, Buenos Aires, Argentina; ${ }^{4}$ SAAO, Cape, South Africa; \\ ${ }^{5}$ Observatorium Hoher List, Universitäts-Sternwarte Bonn, F. R. Germany
}

EZ CMa (HD 50896, WN5) is an enigmatic object. New photometry and polarimetry of EZ CMa are presented in the figure. Again the $\mathbf{3 . 7 7}$ day period is found but, as observed at previous epochs (e.g. Drissen et al. 1989, Ap. J., 343, 426), the shapes of the curves change. The new photometry can also be interpreted in terms of a shorter period, of 1.254 days. A period of about one day is also claimed in other sets of photometric data (e.g. van der Hucht et al., 1990, A. A., 228, 108) and in the IUE spectra of St.-Louis et al. (1990, this symposium). However, despite the complex nature of the light curve, the $\mathbf{3 . 7 7}$ day period is strongly supported by the polarimetry, which shows no evidence for the shorter period.

If EZ CMa is a binary WR + c system (Firmani et al. 1980, Ap. J., 239, 607), then the short-term phase-dependent variations could be an indication of interaction between the WR wind and the 3.77 day orbiting companion. Long term changes may be related to the precession of an accreting disk around the companion; however, no long periodicity is obvious yet. The possibility of a single rotating or pulsating star must also be considered.
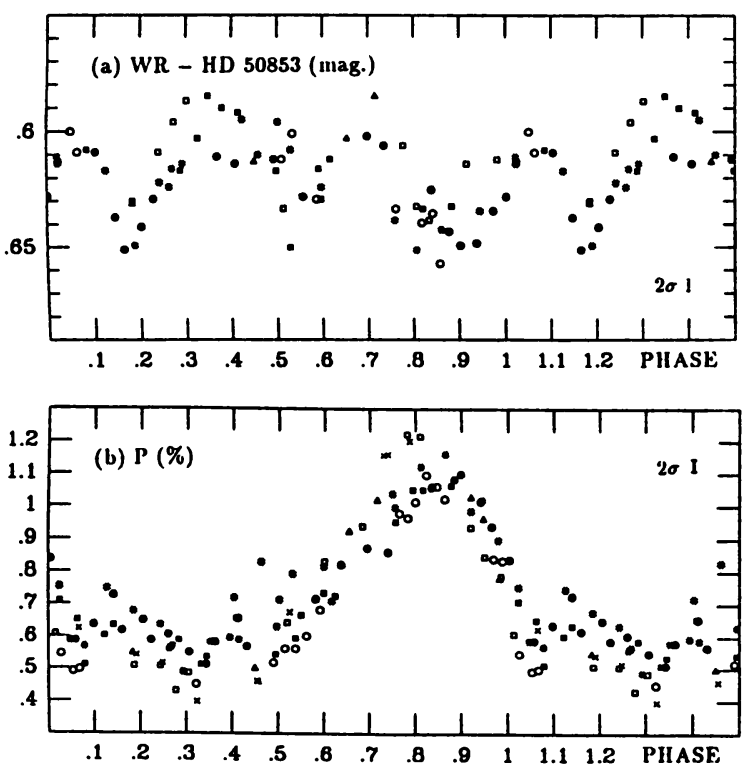

181

FIGURE. (a) Light curve (WR - HD 50853) and (b) polarization curve ( $P$, the degre of polarization) of EZ CMa in 1990. The phase has been calculated from the Lamontagne et al. (1986, Astron. J., 91, 925) ephemeris. Measurements obtained at the San Juan Observatory (Argentina) with VATPOL and a $30 \AA$ wide filter centered on $4700 \AA$ (continuum) are plotted for the observing runs Jan. 13 to Feb. 1 (*) and Mar. 19 to $27(x)$. Filled symbols are data from the University of Toronto Southern Observatory (Chile) collected with MINIPOL and a $G$ filter from Feb. 5 to $17(\bullet), \quad$ Feb. 18 to Mar. 1 $(-)$ and Mar. 17 to $22(\Delta)$. Open symbols refer to the data collected with the South African Astronomical Observatory photo-polarimeter from Feb. 19 to 27 (o) and Mar. 20 to Apr. 1 (o), both with a Johnson $V$ filter. Each group of data has been shifted in ordinate to give the same mean value.

K. A. van der Hucht and B. Hidayat (eds.),

Wolf-Rayet Stars and Interrelations with Other Massive Stars in Galaxies, 181.

(C) 1991 IAU. Printed in the Netherlands. 